\title{
Evolution des apports en eau dans la plaine rizicole de Marovoay par le fleuve Betsiboka (Madagascar) : reconstitution et projections jusqu'en 2100
}

\author{
Dominique Dumas $^{1}$, Sylvain Bigot ${ }^{2}$, Yao Télesphore Brou ${ }^{3}$, Rivo Ramboarison ${ }^{4}$, Samuel Razanaka ${ }^{5}$, and \\ Laurent Roger Robison ${ }^{5}$ \\ ${ }^{1}$ Université Lyon 3 - Environnement Ville Société (EVS, UMR 5600), 69362 Lyon, France \\ ${ }^{2}$ UGA - Institut des Géosciences de l'Environnement (IGE, UMR 5001), 38000 Grenoble, France \\ ${ }^{3}$ Université de La Réunion - ESPACE-DEV (UMR 228), 97744 Saint-Denis, France \\ ${ }^{4}$ Cabinet d'études BEST-IDEES, Antananarivo, Madagascar \\ ${ }^{5}$ Centre National de Recherche en Environnement (CNRE), Antananarivo, Madagascar
}

Correspondence: Dominique Dumas (dominique.dumas@univ-lyon3.fr)

Published: 16 November 2021

\begin{abstract}
Résumé. Aujourd'hui, le fleuve Betsiboka alimente une vaste plaine agricole située au NW de Madagascar. Ce fleuve est pourtant très mal connu sur un plan hydrologique. Sur la base de mesures des écoulements entre 1958 et 1971, cette étude cherche dans un premier temps à reconstituer les débits contemporains de ce fleuve, puis dans un second temps à cerner comment les écoulements peuvent évoluer au cours du XXIème siècle. Les résultats de cette étude montrent qu'une baisse des débits semble se dessiner à la fin de ce siècle. Elle diminuerait la ressource en eau apportée par le fleuve, selon les scénarii climatiques retenus, de 2.4 à $2.7 \mathrm{~km}^{3}$ d'eau par an. Cette diminution de la ressource serait par ailleurs plus marquée sur la période de décembre à février, durant justement la période de repiquage du riz qui implique un apport en eau élevé.
\end{abstract}

\section{Introduction}

La plaine rizicole de Marovoay, avec une superficie de presque 18000 ha de cultures, constitue le deuxième grenier à riz de Madagascar, après la région du lac d'Alaotra. L'alimentation en eau de cette plaine est largement liée au fleuve Betsiboka (Bigot et al., 2018), ainsi qu'à ses affluents, dont les bassins s'inscrivent largement au sein du Parc National d'Ankarafantsika et de son espace forestier (l'une des dernières grandes forêts sèches malgaches). Certains secteurs de la plaine sont d'avantage tributaires directement du fleuve Betsiboka, et d'un apport en eau par pompage de ses eaux. La diminution des écoulements du fleuve et la possibilité de mobiliser des quantités d'eau rendent aujourd'hui l'irrigation plus délicate, voire aléatoire.

A l'inverse, il semblerait que la durée d'inondation, par débordement du fleuve, se soit allongée (Clark, 2012; Lawrence et Vandecar, 2015). Les agriculteurs doivent alors retarder la mise en culture. La période de la campagne cultu- rale est donc non seulement décalée, mais aussi, sur la fin de campagne, raccourcie par le manque d'eau. Les agriculteurs ne peuvent plus compter totalement sur les apports du fleuve. La riziculture pluviale, encore marginale sur l'ensemble de la plaine de Marovoay il y a quelques années, s'étend de plus en plus. Sur ces secteurs, pour des raisons économiques (prix du carburant, vétusté du matériel de pompage), mais aussi probablement hydrologiques (étiages plus longs, excès d'eau au mauvais moment...), les parcelles sont aujourd'hui de plus en plus exploitées à travers une riziculture pluviale, où la maîtrise de l'eau, et donc du calendrier rizicole, n'est dès lors plus possible.

Quelle est la part des raisons consécutives à une irrigation défaillante ou mal maitrisée, et celles liées à des modifications à venir des écoulements du fleuve ? Une gestion efficace de cette plaine rizicole implique nécessairement de pouvoir répondre à cette interrogation et celles qui en découlent. Dans cet objectif, une connaissance des écoulements du fleuve reste indispensable. Or, le réseau hydro- 


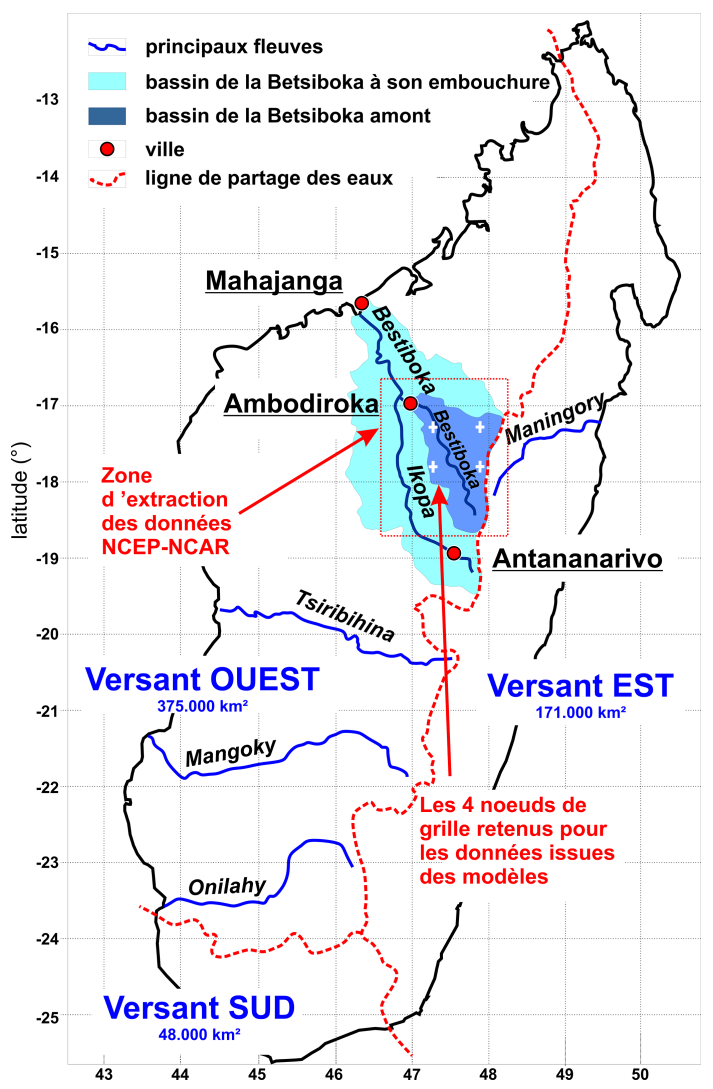

Figure 1. Localisation du bassin de la Betsiboka à Madagascar, et position des extractions des données NCEP-NCAR et des modèles climatiques globaux.

logique malgache, souvent à l'abandon, ne permet plus de fournir des informations suffisantes pour évaluer et cerner les évolutions des écoulements. Par ailleurs, les mesures et observations collectées dans les années 1970-1990 (Chaperon et al., 1993) ne sont plus toujours accessibles au sein des services hydrologiques malgaches, ou dans des bases de données. Certaines données acquises ont été visiblement perdues, malgré les moyens considérables mis en place pour les acquérir dans les années 70 et 80 . Ainsi, sur la Betsiboka, au niveau de son embouchure, où se place la plaine de Marovoay, la connaissance des débits passés et actuels est devenue difficile. Pourtant, après la Mangoky $\left(55700 \mathrm{~km}^{2}\right)$ et la Tsiribihina $\left(49800 \mathrm{~km}^{2}\right)$, la Betsiboka est le troisième fleuve malgache par la taille de son bassin qui collecte des affluents sur plus de $49000 \mathrm{~km}^{2}$.

Au niveau de la plaine de Marovoay, l'apport en eau plus réduit est déjà une réalité ces dernières années par le contexte environnemental modifié, par la déforestation et par la variabilité climatique (Maina et al., 2012; Bigot et al., 2020). Ce travail se propose justement de mieux cerner les écoulements actuels de ce grand fleuve malgache, puis d'évaluer pour l'ensemble du XXIème siècle leurs fluctuations attendues. D'abord, cette étude cherche à reconstruire mensuel- lement les données hydrologiques sur une période récente, pour mieux saisir le comportement hydrologique du fleuve et ses fluctuations actuelles. Puis, à partir de cette modélisation des écoulements, et de données complémentaires issues de modèles climatiques, cette étude montre les évolutions possibles des écoulements de la Betsiboka pour l'ensemble du XXIème siècle.

\section{Données et méthodologie}

\subsection{Reconstitution des écoulements de la Betsiboka à Ambodiroka}

Avec des variations saisonnières marquées, son débit mensuel moyen passe de 120 à $4000 \mathrm{~m}^{3} / \mathrm{s}$, son module est à l'embouchure vraisemblablement proche de $800 \mathrm{~m}^{3} / \mathrm{s}$, dont $460 \mathrm{~m}^{3} / \mathrm{s}$ sont apportés par son affluence principal de l'Ikopa (à partir des données in Chaperon et al., 1993). Notons que ces valeurs semblent éloignées des descriptions observées dans la littérature plaçant son module à $270 \mathrm{~m}^{3} / \mathrm{s}$ (Raharimahefa et Kusky, 2010; Duvail et al., 2017) qui est en fait le module de la Betsiboka avant sa confluence avec l'Ikopa (approché à partir de la station hydrologique d'Ambodiroka). La raison tient probablement à la confusion liée au fait qu'il n'existe pas d'autres stations plus à l'aval, et qu'elle décrit la Betsiboka dans l'ouvrage monographique de référence de Chaperon et al. (1993). Le module y est d'ailleurs évalué plus exactement à $297 \mathrm{~m}^{3} / \mathrm{s}$ sur la période $1958-1973$.

Les écoulements de la Betsiboka ont été mesurés de 1957 à 1983 , et de 1988 à 1993, à la station hydrologique d'Ambodiroka. Son bassin est alors de $11800 \mathrm{~km}^{2}$. Les débits ont été calculés avec une grande précision puisque plus de 150 jaugeages ont été effectués dans une large gamme de débits allant de 40 à $7800 \mathrm{~m}^{3} / \mathrm{s}$ (Chaperon et al., 1993). De 1958 à 1971, les données hydrologiques présentent peu de lacunes, contrairement à celles inscrites après 1971, et plus encore entre 1988 et 1993. Les données après l'année 1971 sont ainsi écartées de cette étude par leur mauvaise qualité.

Si dans les années 60, on recensait plus de 250 stations météorologiques, aujourd'hui les stations sont peu nombreuses, et par ailleurs se placent pour l'essentiel sur le littoral ou à proximité de la capitale (Bigot et al., 2018). A partir des produits du National Centers for Environmental Prediction - National Center for Atmospheric Research (NCEP-NCAR), il est possible d'obtenir les données journalières de réanalyses atmosphériques de 1958 à 2020 (Fig. 1) avec une résolution spatiale de $1.875^{\circ}$ (Kalnay et al., 1996; Grist et Nicholson, 2001). Sur la période 1950 à 2020, il est dès lors possible d'examiner les variations quotidiennes moyennes des pluies et des températures sur une zone intégrant le bassin de la Betsiboka à Ambodiroka. Dans ce travail, nous utiliserons cet ensemble de données NCEP-NCAR pour calculer l'évaporation potentielle puis calculer les écoulements à partir d'un modèle hydrologique conceptuel global (GR2M). 
L'évaporation potentielle sur le bassin est appréciée à partir de la température journalière des réanalyses NCEPNCAR. L'introduction du rayonnement extraterrestre journalier $\left(R e_{j}\right)$, calculé ici pour la latitude moyenne du bassin de $-17.5^{\circ} \mathrm{S}$ permet d'affiner l'estimation de l'évaporation potentielle (Oudin, 2004; Oudin et al., 2005) (Eq. 1).

$\operatorname{ETP}_{j}=\frac{R e_{j}}{\gamma \rho} \frac{T_{j}+5}{100}$

avec $\gamma$, la chaleur latente de vaporisation de l'eau $\left(2,25 \mathrm{MJ} \mathrm{kg}^{-1}\right) ; \quad \rho$, la masse volumique de l'eau $\left(1000 \mathrm{~kg} \mathrm{~m}^{-3}\right) ; \quad R e_{j}$, le rayonnement extraterrestre $\left(\mathrm{MJ} \mathrm{m}^{-2} \mathrm{j}^{-1}\right)$ calculé à la latitude $-17.5^{\circ}$ et $\mathrm{du}$ jour julien $j ; T_{j}$, la température moyenne du jour julien $j\left({ }^{\circ} \mathrm{C}\right)$.

L'estimation des écoulements mensuels utilise le modèle conceptuel global GR2M (Génie Rural Mensuel à deux paramètres), dont la description est détaillée par de nombreux travaux (Mouelhi et al., 2006, 2013). Il est constitué de deux réservoirs, que l'on alimente avec des données mensuelles de pluie et d'évaporation potentielle, et par deux coefficients qui caractérisent le dimensionnement et le fonctionnement de ces réservoirs. Ces deux paramètres caractérisent dès lors le fonctionnement hydrologique de l'ensemble du bassin versant. Ils sont définis par comparaison avec les débits mesurés - pour la Betsiboka de 1958 à 1971 - par itération avec le critère d'optimisation de Nash-Sutcliffe (Fig. 2, Éq. 2). Cet indicateur juge de la qualité de l'estimation des débits modélisés par rapport à des débits observés (Nash et Sutcliffe, 1970; Perrin et al., 2003). La valeur de cet indice varie entre $-\infty$ et $100 \%$, et se rapproche de $100 \%$ lorsque les débits calculés se calent sur les débits observés. L'équation suivante le définit comme :

$\mathrm{NS}(\%)=100\left[1-\frac{\sum\left(Q_{\mathrm{obs}}-Q_{\mathrm{cal}}\right)^{2}}{\sum\left(Q_{\mathrm{obs}}-\bar{Q}_{\mathrm{obs}}\right)^{2}}\right]$

L'indice de Nash est de $71 \%$ dans cette reconstitution. Au regard de la sévérité de cet indicateur, une performance supérieure à $60 \%$ est généralement jugée satisfaisante dans les modèles hydrologiques (Perrin et al., 2003).

\subsection{Estimations des pluies et des températures mensuelles jusqu'en 2100}

Les projections climatiques de 7 modèles climatiques globaux, calculées mensuellement par vingtaines jusqu'en 2100, et sur 4 points de grille (Fig. 1, Tableau 1), ont été utilisées. Pour les données de projections climatiques futures, cette étude a utilisé deux scénarii d'émission RCP (Representative Concentration Pathways); le scénario moyen RCP4.5, et celui, plus pessimiste, RCP8.5 supposant un forçage élevé dans l'émission des gaz à effet de serre. Le choix d'utiliser des moyennes calculées sur des périodes de 20 ans a permis de s'affranchir de la variabilité interannuelle pour ne conserver que les principales modifications tendancielles. Cette ap- proche multi-modèle a limité également le poids et l'incertitude liés aux résultats issus d'un unique modèle.

Le Tableau 1 synthétise les moyennes annuelles des sorties des 7 modèles climatiques pour les deux scénarii retenus. On observe naturellement des différences entre les modèles pour les deux paramètres climatiques, même si les variabilités sont plus marquées pour les précipitations. La moyenne des 7 modèles de projection montre une hausse des températures sur l'ensemble du XXIème siècle serait de $1.7^{\circ} \mathrm{C}$ dans le cas du scénario RCP4.5, et de $4.4^{\circ} \mathrm{C}$ pour le scénario RCP8.5. En revanche, et quel que soit le scénario, les pluies n'indiquent pas d'évolution simple au cours du XXIème siècle.

\section{Résultats et discussion}

\subsection{Reconstitution des écoulements mensuels de 1950 à 2019}

Sur la période 1958 à 1971, et à partir des températures et précipitations journalières issues des réanalyses NCEPNCAR, il est dès lors possible de caler le modèle GR2M. La performance obtenue, de $71.1 \%$, montre qu'il est possible de reconstituer les débits à partir de ces données à une relative robustesse (Fig. 2). Dès lors, il est possible de calculer les débits sur l'ensemble de la période 1958-2019 pour laquelle les températures et précipitations sont connues mensuellement à partir des réanalyses NCEP-NCAR. Les débits mensuels moyens par vingtaine peuvent, par exemple, être précisés (Tableau 2).

\subsection{Diminution des écoulements sur l'ensemble du XXlème siècle}

Le Tableau 3 montre les modules de la Betsiboka par vingtaines allant de 2020 à 2099. Les valeurs avant 2019 sont obtenues à partir des données de réanalyses, après, les valeurs des écoulements sont calculées à partir des modèles climatiques globaux selon les deux scénarii RCP retenus.

L'utilisation de deux types de données climatiques, et sur des espaces différents, produit un biais dans la reconstitution des écoulements entre les vingtaines 2000-2019 et 20202039 (Tableau 3). Il implique de prendre les valeurs absolues estimées avec prudence. En revanche, l'évolution générale des débits est plus signifiante, et plus encore si on ne regarde que les projections hydro-climatiques jusqu'en 2100 .

Les écoulements calculés varient naturellement fortement avec les modèles utilisés. Par exemple, sur la période 2060 à 2079, avec le scénario RCP4.5, les modules reconstitués de la Betsiboka peuvent varier de 187 à $359 \mathrm{~m}^{3} \mathrm{~s}^{-1}$ selon le modèle retenu. Curieusement, l'analyse des modules par vingtaine montre des valeurs très proches quel que soit le scénario RCP retenu, les moyennes des débits des 9 modèles sont quasiment identiques sur toutes les vingtaines.

Ces évolutions illustrent sans doute l'aspect systémique du contexte climatique, toute modification d'un paramètre a des 


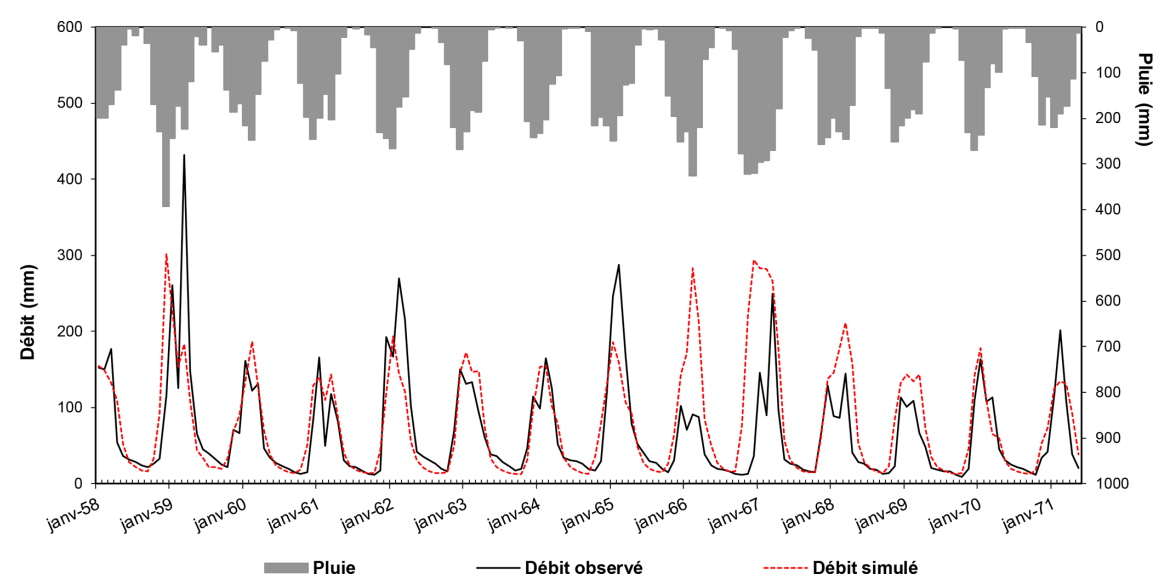

Figure 2. Modélisation des débits mensuels de la Betsiboka à Ambodiroka à partir du modèle hydrologique CR2M, et débits mesurés de 1958 à 1971 .

conséquences sur d'autres. Ici, l'augmentation plus forte des températures (dans le cas du scénario RCP8.5), et donc de l'évaporation, est compensée par des pluies accrues. Mais à l'échelle de ce petit bassin, cette évolution aussi équilibrée n'était pas forcément imaginable au départ. Les tendances des évolutions des écoulements restent les mêmes, une baisse moyenne de $140 \mathrm{~m}^{3} \mathrm{~s}^{-1} 100 \mathrm{ans}^{-1}$ dans le cas du scénario RCP4.5, contre $125 \mathrm{~m}^{3} \mathrm{~s}^{-1} 100$ ans $^{-1}$ pour le scénario RCP8.5. Le scénario le plus pessimiste montre une baisse légèrement plus réduite des écoulements que celui plus optimiste. La diminution moyenne des débits entre 2020 et 2100 serait respectivement de 86 et $77 \mathrm{~m}^{3} \mathrm{~s}^{-1}$ selon les scénarii, soit une perte annuelle de la ressource en eau de 2.4 à $2.7 \mathrm{~km}^{3}$, ce qui est considérable.

Pour les prochaines décennies (2020-2100), à l'échelle mensuelle (Tableau 4), les modifications de l'écoulement de la Betsiboka apparaissent plus contrastées, parfois imperceptibles, parfois très marquées. A l'échelle mensuelle, les modifications restent aussi relativement similaires quel que soit les scénarii RCP retenus. La diminution de l'écoulement mensuel, abordée à partir de la moyenne des 7 modèles climatiques globaux, montre clairement une intensité plus marquée en décembre, janvier et février. La diminution moyenne tendancielle sur l'ensemble de la période étudiée serait même en janvier de $957 \mathrm{~m}^{3} \mathrm{~s}^{-1} 100 \mathrm{ans}^{-1}$ à $914 \mathrm{~m}^{3} \mathrm{~s}^{-1} 100 \mathrm{ans}^{-1}$ selon les scénarii RCP. En période de basses eaux, de juin à novembre, les écoulements ne sont que légèrement modifiés.

\section{Conclusions}

Les reconstitutions des débits mensuels avec des données climatiques NCEP-NCAR produisent des résultats satisfaisants (Nash $=71 \%$ ). Avec l'introduction des projections climatiques issues des modèles globaux, la modélisation hydroclimatique offre alors la possibilité de déceler les grandes tendances hydrologiques pour les prochaines décennies. Sur la base de plusieurs projections climatiques issues de diffé- rents modèles numériques, l'étude des débits de la Betsiboka indique que les écoulements risquent de diminuer ces prochaines décennies. Curieusement, quel que soit l'hypothèse d'émission envisagée, la baisse des ressources en eau semble relativement identique, l'accroissement du prélèvement par évaporation, dans le cas du scénario RCP8.5, est compensé par une majoration des précipitations. A l'échelle saisonnière, la baisse des ressources en eau est fortement contrastée selon les mois. Elle est très marquée en janvier, et forte en décembre et février.

Au niveau de la plaine de Marovoay, l'apport en eau plus réduit est déjà une réalité ces dernières années par le contexte environnemental modifié, par la déforestation et par la variabilité climatique (Maina et al., 2012; Bigot et al., 2020). Ces projections hydro-climatiques sont néanmoins inquiétantes, et montrent que sur la fin de ce siècle, la diminution de l'écoulement sera probablement guidée en premier lieu par le changement climatique. Cette baisse de la ressource en eau ne sera pas sans conséquence sur les pratiques agricoles de la région. La première campagne de riz notamment risque d'être fortement menacée ces prochaines années. Pour cette campagne, la période de repiquage se place de novembre à janvier, et implique un apport en eau élevé, or elle coïncidera tout particulièrement à une baisse très forte des écoulements. En revanche, la seconde campagne de riz, de contre-saison, avec un repiquage d'avril à juin, sera a priori moins affectée par cette évolution hydro-climatique. Dans des perspectives de projets d'aménagement hydro-agricole, les gestionnaires du fleuve, ou ceux de la plaine de Marovoay, doivent pouvoir s'appuyer sur les dynamiques hydrologiques actuelles, mais également sur des projections au cours du XXIème siècle.

Disponibilité du code. Le modèle GR2M peut être utilisé à partir de feuilles Excel : https://webgr.inrae.fr/modeles/mensuel-gr2m/ (Perrin et Andréassian, 2021). 
Disponibilité des données. Données hydrologiques : https:// www.documentation.ird.fr/hor/fdi:37307 Chaperon et al. (1993). Les données NCEP-NCAR : https://psl.noaa.gov/data/gridded/data. ncep.reanalysis.html (National Centers for Environmental Prediction et al., 1994).

Collaborateurs. DD a réalisé la conceptualisation de la méthode, le traitement et l'écriture du manuscrit. Les co-auteurs ont contribué comme suit : SB et TYB ont examiné les données NCEP-NCAR, examiné le processus de rédaction et contribué à la version finale du manuscrit; RR, SR et LRR ont participé à l'acquisition des données, et examiné le processus de rédaction.

Intérêts concurrents. Les auteurs déclarent qu'ils n'ont aucun conflit d'intérêts.

Clause de non-responsabilité. Publisher's note : Copernicus Publications remains neutral with regard to jurisdictional claims in published maps and institutional affiliations.

Déclaration du numéro spécial. This article is part of the special issue "Hydrology of Large River Basins of Africa". It is a result of the 4th International Conference on the "Hydrology of the Great Rivers of Africa", Cotonou, Benin, 13-20 November 2021.

Remerciements. Les auteurs remercient les relecteurs anonymes pour leurs remarques qui ont contribué à améliorer ce manuscrit.

\section{Références}

Bigot, S., Dumas, D., Brou, Y. T., Ramboarison, R., Razanaka, S., Descroix, L., Philippon, N., and Gwiazdzinski, L. : La difficulté du monitoring climatique à une échelle fine dans une région fortement impactée par la déforestation tropicale : la forêt sèche du parc national d'Ankarafantsika (Madagascar), Publications de l'Association Internationale de Climatologie, 31, 356-361, 2018.

Bigot, S., Dumas, D., Brou, T., Ramboarison, R., Razanaka, S., and Philippon, N. : Etude hydroclimatique dans le nord-ouest de Madagascar : apport des données satellitaires GRACE, Publications de l'Association Internationale de Climatologie, 33, 121-126, 2020.

Chaperon, P., Danloux, J., and Ferry, L. : Fleuves et rivières de Madagascar, Monographies hydrologiques, 10, ORSTOM, Paris, France, 874 pp., 1993.

Clark, M. : Deforestation in Madagascar : Consequences of population growth and unsustainable agricultural processes, Global Majority E-Journal, 3, 61-71, 2012.

Duvail, S., Hamerlynck, O., Paron, P., Hervé, D., Nyingi, W. D., and Leone, M. : The changing hydro-ecological dynamics of rivers and deltas of the Western Indian Ocean : Anthropogenic and environmental drivers, local adaptation and policy response, C. R. Geosci., 349, 269-279, https://doi.org/10.1016/j.crte.2017.09, 2017.
Grist, J. P. and Nicholson, S. E. : A study of the dynamic factors influencing the rainfall variability in the West African Sahel, J. Climate, 14, 1337-1359, https://doi.org/10.1175/15200442(2001)014<1337 :ASOTDF>2.0.CO2, 2001.

Kalnay, E., Kanamitsu, M., Kistler, R., Collins, W., Deaven, D., Gandin, L., and Zhu, Y. : The NCEP/NCAR 40-year reanalysis project, B. Am. Meteorol. Soc., 77, 437-472, https://doi.org/10.1175/15200477(1996)077<0437 :TNYRP>2.0.CO2, 1996.

Lawrence, D. and Vandecar, K. : Effects of tropical deforestation on climate and agriculture, Nat. Clim. Change, 5, 27-36, https://doi.org/10.1007/s00382-014-2203-8, 2015.

Maina, J., de Moel, H., Vermaat, J. E., Bruggemann, J. H., Guillaume, M. M., Grove, C. A., Madin, J. S., Mertz-Kraus, R., and Zinke, J. : Linking coral river runoff proxies with climate variability, hydrology and land-use in Madagascar catchments, Mar. Pollut. Bull., 64, 2047-2059, 2012.

Mouelhi, S., Michel, C., Perrin, C., and Andréassian, V. : Stepwise development of a two-parameter monthly water balance model, J. Hydrol., 318, 200-214, https://doi.org/10.1016/j.jhydrol.2005.12.022, 2006.

Mouelhi, S., Madani, K., and Lebdi, F. : A structural overview through GR (s) models characteristics for better yearly runoff simulation, Open Journal of Modern Hydrology, 318, 200-214, https://doi.org/10.1016/j.jhydrol.2005.12.022, 2013.

Nash, J. E. and Sutcliffe, J. V. : River flow forecasting through conceptual models Part one : A discussion of principles, J. Hydrol., 27, 282-290, https://doi.org/10.1016/00221694(70)90255-6, 1970 .

National Centers for Environmental Prediction/National Weather Service/NOAA/U.S. Department of Commerce : updated monthly, NCEP/NCAR Global Reanalysis Products, 1948continuing, Research Data Archive at NOAA/PSL, accessible à l'adresse suivante : https://psl.noaa.gov/data/gridded/data.ncep. reanalysis.html (la date du dernier accès : juin 2021), 1994.

Oudin, L. : Recherche d'un modèle d'évapotranspiration potentielle pertinent comme entrée d'un modèle pluie-débit global, Sciences of the Universe, ENGREF (AgroParisTech), 2004.

Oudin, L., Hervieu, F., Michel, C., Perrin, C., Andréassian, V., Anctil, F., and Loumagne, C. : Which potential evapotranspiration input for a lumped rainfall-runoff model ? : Part 2 - Towards a simple and efficient potential evapotranspiration model for rainfall-runoff modelling, J. Hydrol., 303, 290-306, https://doi.org/10.1016/j.jhydrol.2004.08.026, 2005.

Perrin, C. et Andréassian, V. : Á partir du modèle mise au point par Mouelhi (2003), Application du modèle pluie-débit mensuel GR2M sous Excel, accessible à l'adresse suivante : https: //webgr.inrae.fr/modeles/mensuel-gr $2 \mathrm{~m} /$, la date du dernier accès : octobre 2021.

Perrin, C., Michel, C., and Andréassian, V. : Improvement of a parsimonious model for streamflow simulation, J. Hydrol., 279, 275289, https://doi.org/10.1016/j.jhydrol.2004.08.026, 2003.

Raharimahefa, T. and Kusky, T. M. : Environmental monitoring of Bombetoka Bay and the Betsiboka Estuary, Madagascar, using multi-temporal satellite data, J. Earth Sci., 21, 210-226, https://doi.org/10.1007/s12583-010-0019-y, 2010. 